\title{
Neuroimaging in contact sports: Determining brain fitness before and after a bout
}

\author{
N K Sethi, MD \\ Department of Neurology, New York Presbyterian Hospital, Weill Cornell Medical Center, New York, USA
}

Corresponding author: N K Sethi (sethinitinmd@hotmail.com)

Background: Professional boxing and mixed martial arts (MMA) are popular contact sports with high risk for both acute and chronic traumatic brain injury (TBI). Although rare, combatants have died in the ring/cage or soon after the completion of the bout. The cause of death in these cases is usually acute subdural hematoma, acute epidural hematoma, subarachnoid haemorrhage, intracranial haemorrhage, or second-impact syndrome (SIS). Neuroimaging or brain imaging is currently included in the process of registering for a license to fight in a combat sport in most states in the United States and around the world. However, the required imaging specifics and frequency vary.

Discussion: Neuroimaging serves two distinct roles in the individualised care of a combatant, representing a step towards personalised medicine and individual risk stratification. Neuroimaging prior to licensure helps to identify and/or exclude coincidental or clinically suspected brain lesions which may pose a risk for rupture, bleeding or other catastrophic and important brain injury. Neuroimaging in the immediate aftermath of a bout primarily serves to rule out acute traumatic brain injury. Neuroimaging may also be carried out to assess for evidence of structural brain injury which may make a combatant more likely to express late-life neuropsychiatric sequelae of brain injury, such as chronic traumatic encephalopathy. As such, neuroimaging plays a prognostic role and aids in the determination of whether the combatant should be allowed to continue to participate in future bouts or not.

Conclusion: Currently there are no established neuroimaging guidelines for contact sports. Standardising neuroimaging guidelines both for licensure as well as neuroimaging modality, and protocols to assess for both acute and chronic traumatic brain injury. This will assist in protecting the combatant's health and safety, both in the ring/cage, and after their professional careers have ended. Some suggested guidelines are provided based on currently available medical literature. It is recommended that these guidelines be debated vigorously by the scientific community and that evidence-based guidelines be developed by the medical community in conjunction with professional boxing and MMA governing bodies.

Keywords: boxing, MMA, traumatic brain injury, chronic traumatic encephalopathy

S Afr J Sports Med 2017;29:1-4.DOI: 10.17159/2078-516X/2017/v29i0a2390

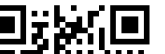

The risks for both acute and chronic traumatic brain injury are high in contact sports such as professional boxing and MMA. Brain imaging 口ifter either computed tomography (CT) or magnetic
resonance imaging (MRI), is currently included in the process of registering for a license to fight in combat sports. The imaging requirements for licensure, though vary. Some state commissions in the United States require an MRI brain scan every three years, others once, at the time of licensure, and some do not require any imaging prior to licensure. Brain imaging, particularly a CT scan of the head is currently the imaging modality of choice to rule out acute traumatic brain injury after a bout. There are no guidelines regarding the imaging for chronic traumatic brain injury and chronic traumatic encephalopathy.

\section{Neuroimaging prior to licensure}

Neuroimaging prior to licensure helps in the guiding of the clinical judgement of supervisory personnel (ringside physicians) regarding whether the combatant should participate in future bouts. It also helps to identify and/or exclude coincidental or clinically suspected brain lesions which may pose a risk for rupture, bleeding or other catastrophic and other injuries to the brain injury should a combatant participate in future bouts. These structural lesions include but are not limited to cerebral aneurysms, arteriovenous malformations, large cavernous malformations, mixed malformations, vein of Galen malformations, large venous malformations, large arachnoid cysts, posterior fossa arachnoid cysts, pituitary macroadenomas and other space-occupying lesions, such as large tumours. It is important to emphasise that the above represents a fairly heterogeneous group of cerebral and cerebrovascular lesions which demonstrate different natural histories and a propensity to bleed. A less urgent but by no means less important role of neuroimaging prior to licensure is to identify any evidence of prior structural injury possibly or probably associated specifically with brain trauma, which may make a combatant more likely to express late-life neuropsychiatric sequelae of brain injury such as chronic traumatic encephalopathy (CTE), dementia pugilistica, chronic post-concussion syndrome, chronic neurocognitive impairment, posttraumatic dementia, posttraumatic cognitive impairment, posttraumatic parkinsonism and persistent posttraumatic headache. [1, 2] This information allows the physician and combatant to make decisions about the combatant's future health, particularly when some of the findings may be ambiguous either in their appearance or in the implication for and risk of future neurologic sequelae.

Either a CT or MRI is currently included in the process of registering for a license to fight in combat sports in most states in the United States and around the world. [3] The imaging specifics and frequency varies widely throughout U.S. states, with some state commissions requiring a MRI brain scan every three years and others only once, i.e. at the time of licensure. Some state commissions do not require any imaging prior to licensure, while New York details the required imaging sequences (Table 1). Some commissions require that all 
Table 1. Imaging requirements for licensure for professional boxing and MMA of various commissions in the United States and around the world

\begin{tabular}{cl}
\hline US State & \multicolumn{1}{c}{ Imaging requirements } \\
\hline Alabama (AL) & Radiological exams: None at this time. \\
& Neurological exam: Any applicant that is more than 37 years of age and/or has competed in more than 200 professional rounds \\
& must submit a detailed neurological examination conducted by a state licensed neurologist.
\end{tabular}

Arizona (AZ) Radiological exams: None at this time.

Neurological exam: Not at this time.

Arkansas (AR) Radiological exams: None at this time.

Neurological exam: Not at this time.

California (CA)

Radiological exams: An MRI of the brain without contrast and the GRE Technique results must also be included. The MRI scan is to be performed on a 1.5 Tesla MR Machine with capabilities including fast spin echo and FLAIR imaging. Image sequences should include axial T1, T2, and FLAIR images; coronal images should be performed as a T2 coronal; and a single sagittal T1 sequence. The images should be placed on a CD as soon as possible and forwarded to the commission at: 2005 Evergreen St., Suite 2010, Sacramento, CA, 95815. If a CD is not available, please forward the actual film within ten (10) days to avoid suspension of the license. The MRI is valid for five years.

Neurological exam: A Neurological exam is required and is valid for 15 months

Colorado (CO) Radiological exams: None at this time.

Neurological exam: Not at this time.

Connecticut (CT) Radiological exams: It is the fighter's choice of an MRI, CT scan, or Neurological exam. All tests/exams are good for three to four years from the date of the fight.

Neurological exam: It is the fighter's choice of an MRI, CT scan, or Neurological exam. All tests/exams are good for three to four years from the date of the fight.

Florida (FL)

Radiological exams: None at this time.

Neurological exam: Not at this time

Delaware (DE) Radiological exams: Fighters who have not fought within the last 16 months or have had five or more knockouts must submit a CT scan administered by a licensed MD or DO.

Neurological exam: Fighters who have not fought within the last 16 months or have had five or more knockouts must submit a CT scan administered by a licensed MD or DO.

Georgia (GA) Radiological exams: None at this time

Neurological exams: Not at this time. Changes will be made in the near future.

Nevada (NV) Radiological exams: Must submit an MRI or MRA of the brain without contrast. This exam is a one-time requirement, unless otherwise ordered.

Neurological exam: Not at this time.

New Jersey (NJ) Radiological exams: CT or MRI of the brain without contrast dated within three years of the event is required.

Neurological exam: Not at this time.

Maryland (MD) Radiological exams: None at this time.

Neurological exam: Boxers must take a neurological exam and it must be completed prior to the boxer participating in his/her first bout (Paid for and chosen by the commission).

Oklahoma (OK) Radiological exams: None at this time.

Neurological exam: Not at this time.

Illinois (IL) Radiological exams: None at this time.

Neurological exam: Not at this time.

Hawaii (HI) Radiological exams: None at this time.

Neurological exam: Not at this time.

Additional requirements: Any fighter that has fought 120 rounds or has not competed within the last 36 months may be subject to additional tests/exams as determined by a licensed physician. All these additional tests/exams will be valid for one year.

New York (NY)

Radiological exams: MRI brain 1.5 Tesla (minimum) or 3 Tesla magnet. Required MRI sequences: T1, T2, FLAIR, diffusion weighted image (DWI), gradient echo (GRE) or susceptibility weight imaging (SWI) - every three years or as required by the commission

Neurological exam: Not at this time. 
combatants undergo a magnetic resonance angiogram (MRA) of the brain at the start of their professional career (time of licensure) primarily to exclude any incidental vascular malformations of the brain which have a high propensity to bleed (Table 1).

The MRI is generally considered to be superior to a CT for detecting the stigmata of chronic TBI. Although the CT is better at detecting bony pathology, some studies have shown that it may have missed between $10-20 \%$ of abnormalities seen on an MRI. ${ }^{[3,4]}$ The ability of the MRI to detect hematomas improves over time as the composition of the blood changes. The overwhelming majority of patients with mild brain injury shows no abnormality on an MRI, but coincidental structural brain abnormalities are found in about $2-3 \%$ of all studies, including meningiomas, coincidental and non-pathologic anomalies, and small often static cerebral aneurysms. Cerebral vasculature anomalies are particularly frequent in the Circle of Willis. With reference to contact sports, relevant abnormalities include hemorrhagic cortical contusions, petechiae, or foci of altered signal that represent white matter shear injury. ${ }^{[5]}$ When petechiae resolve, they leave a permanent hemosiderin deposition on the MRI. An MRI is superior to a CT in the detection of axonal injury, small areas of contusion, and subtle neuronal damage. Stigmata of old TBI on an MRI also include areas of encephalomalacia and gliosis, particularly in areas prone to coup-contrecoup forces, such as the frontal lobe (anterior as well as inferior margin along the cribiform plate), anterior temporal lobe, and the occipital pole. The MRI is also superior to a CT at imaging the brainstem, basal ganglia, and thalami. Fluid-attenuated inversion recovery (FLAIR) MRI, a sequence that suppresses the high signal from cerebrospinal fluid (CSF) by using a long inversion time (T1), has been found to be more sensitive in detecting traumatic lesions and hematomas. White matter abnormalities may be seen on an MRI in patients with mild TBI. However, it should be remembered that white matter changes are also found in a large percentage of healthy middle-aged individuals, middle-aged persons with chronic vascular risk factors including hypertension, as well as younger patients with migraines. ${ }^{[2]}$ Newer MRI technology and acquisition sequences have improved the sensitivity of the MRI for detecting the stigmata of TBI, but not all sequences are routinely adopted or performed in clinical contexts and some are presently strictly related to research. ${ }^{[5,6]}$ Among these in particular is diffusion tensor imaging (DTI); traumatic axonal injury which is characterised by a reduction in fractional anisotrophy (FA) on DTI. [7] Magnetisation transfer imaging (MTI), which applies radio frequency power only to the protons in the macromolecules of tissues rather than the protons in water, can add sensitivity to an MRI. Magnetic source imaging (MSI), using a combination of MRI and magnetoencephalography (MEG), was found to be superior to using only an MRI in the detection of TBI. Proton magnetic resonance spectroscopic imaging (1H-MRSI) has been found to be a sensitive tool in detecting axonal injury in the corpus callosum of TBI patients. Functional MRI can demonstrate changes in regional brain activation in patients with mild TBI. Susceptibility-weighted imaging (SWI) and arterial spin labelling (ASL) are newer MRI methods for better detection of TBI. ${ }^{6]}$

There are presently no good biomarkers (biofluids or imaging) for chronic TBI and its reported link with chronic traumatic encephalopathy (CTE). [8] Multimodal imaging techniques may help to enhance the yield of detection and characterisation of stigmata of prior TBI. Along with neuropsychological testing, these methods may help in the prediction of injuries to fighters who partake in contact sports by providing information of neural networks that subserve cognition and executive function. [9] It is hoped that with advances in neuroimaging and the development of imaging and biofluid biomarkers for TBI and CTE will lead to the development of rational and evidence-based medicine approaches to the management and risk stratification of fighters who demonstrate evidence of prior TBI on their MRI scans.

Recommended good practice guidelines regarding neuroimaging requirements prior to licensure:

1. To ensure the health and safety of all combat sports participants, it is recommended that neuroimaging requirements prior to licensure be standardised across different commissions and sports governing bodies in all countries. Standardisation of neuroimaging requirements shall allow the physician and combatant to take measured decisions about the combatant's future health and brain fitness to begin fighting or continue to fight.

2. It is recommended that the various commissions and sports governing bodies coordinate to assist with the setting up of an online central neuroimaging database so that neuroimaging data can be shared in the different countries where the fighter may fight.

3. A brain MRI is the recommended imaging modality of choice to assess brain fitness to fight as well as to predict possible long-term effects of multiple hits to the head and assess stigmata of chronic TBI.

4. When a brain MRI is not feasible, either due to its nonavailability or if the cost is prohibitive, a CT brain scan may suffice with the knowledge of the limitations of CT scan technology as documented above.

5. The decision to medically clear a fighter to fight when the brain MRI suggests evidence of prior/chronic TBI should be made on a case-by-case basis after careful consideration and review of the fighter's medical history, age, fight record, neurological examination and neurocognitive performance. Ringside physicians may request additional neuroimaging (as detailed below) and a formal neuropsychological evaluation of a fighter.

6. Risk stratification is the process of identifying the individual risk of a combatant suffering from a particular condition after careful review of history, laboratory and clinical tests. Some combatants may warrant disqualification and denial of license to fight and others may be medically cleared to fight after careful review of all relevant history and imaging findings as mentioned above.

\section{Neuroimaging after a bout}

The prime need for neuroimaging after a bout is to ascertain acute TBI. A CT scan of the head without contrast is the recommended imaging modality due to its relatively high sensitivity and specificity for identifying the presence of blood in the acute setting, its widespread availability and short acquisition time. 
Recommended good practice guidelines regarding neuroimaging requirements after a bout:

1. Based on the assessment by the ringside physician(s) after the post-fight physical examination, a combatant who is suspected of having sustained a TBI should be transported immediately by ambulance to the designated Level I trauma centre for an emergency assessment of possible TBI/concussion, an urgent CT scan of the head, and further care as deemed necessary by the trauma centre physicians. Concern for TBI is raised if the combatant manifests or reports symptoms of headache, blurred vision, double vision, nausea, vomiting, balance or gait issues after a bout. [10,11]

2. Any combatant with a Glasgow Coma Scale/Score (GCS) of less than 13 on initial assessment, suspected open or depressed skull fracture, suspected basal skull fracture, post-traumatic seizure, focal neurological deficit and/or greater than one episode of vomiting since the suspected head injury should be urgently transported to the designated Level I trauma centre via an on-site ambulance for a CT scan of the head (as per National Institutes of Health and Care Excellence (NICE) guidelines for determining the need for an acute CT scan of the head in adults following a traumatic head injury). [12]

\section{Conclusion}

It is recommended that the best practice guidelines as mentioned above be debated vigorously by ringside physicians and the larger scientific community. The evidence-based guidelines on neuroimaging requirements prior to licensure, in the immediate post-bout setting, and the assessment for the stigmata of chronic TBI and CTE be investigated by the medical community in conjunction with professional boxing and MMA governing bodies. The establishment of these guidelines will go a long way in ensuring the health and safety of professional combatants.

Author contributions: NKS conceived, drafted and revised the manuscript.
Study funding: No targeted funding reported.

Disclosure: NKS serves as Associate Editor, The Eastern Journal of Medicine and as Chief Medical Officer of the New York State Athletic Commission (NYSAC). The views expressed are his and do not represent the views of the NYSAC.

\section{References}

1. Ling H, Hardy J, Zetterberg H. Neurological consequences of traumatic brain injuries in sports. Mol Cell Neurosci 2015; 66(Pt B):114122. [doi: 10.1016/j.mcn.2015.03.012]

2. Seifert $T$, Bernick $C$, Jordan $B$, et al. Determining brain fitness to fight: Has the time come? Phys Sportsmed. 2015; 43:395-402. [doi: 10.1080/00913847.2015.1081551]

3. Jordan BD, Jahre C, Hauser WA, et al. CT of 338 active professional boxers. Radiology 1992; 185:509-512. [doi: 10.1148/radiology.185.2.1410364]

4. Lee B, Newberg A. Neuroimaging in traumatic brain imaging. NeuroRx 2005; 2: 372-383. [doi: 10.1602/neurorx.2.2.372]

5. Koerte IK, Hufschmidt J, Muehlmann M, et al Advanced neuroimaging of mild traumatic brain injury. In: Laskowitz D, Grant G, eds. Translational Research in Traumatic Brain Injury. Boca Raton (FL): CRC Press/Taylor and Francis Group; 2016. Chapter 13.

6. Van Boven RW, Harrington GS, Hackney DB,et al. Advances in neuroimaging of traumatic brain injury and posttraumatic stress disorder. J Rehabil Res Dev 2009; 46:717-757. [PMCID: PMC3233771]

7. Li L, Sun G, Liu K,et al. White matter changes in posttraumatic stress disorder following mild traumatic brain injury: A prospective longitudinal diffusion tensor imaging study. Chin Med J (Engl) 2016; 129:1091-1099. [doi: 10.4103/0366-6999.180518]

8. Papa L, Ramia MM, Edwards D, et al. Systematic review of clinical studies examining biomarkers of brain injury in athletes after sports-related concussion. J Neurotrauma 2015; 32:661-673. [doi: 10.1089/neu.2014.3655]

9. Multani N, Goswami R, Khodadadi $\mathrm{M}$, et al. The association between white-matter tract abnormalities, and neuropsychiatric and cognitive symptoms in retired professional football players with multiple concussions. J Neurol 2016; 263:1332-1341. [doi: 10.1007/s00415-016-81410]

10. National Clinical Guideline Centre (UK). Head Injury: Triage, Assessment, Investigation and Early Management of Head Injury in Children, Young People and Adults. London: National Institute for Health and Care Excellence (UK); 2014 Jan [PMID: 25340248]

11. Davis T, Ings A, National Institute of Health and Care Excellence Head injury: triage, assessment, investigation and early management of head injury in children, young people and adults (NICE guideline CG 176). Arch Dis Child Educ Pract Ed 2015; 100:97-100. [doi 10.1136/archdischild-2014-306797]

12. Hodgkinson S, Pollit V, Sharpin C, et al. Early management of head injury: summary of updated NICE guidance. BMJ 2014; 348:g104. [doi 10.1136/bmj.g104] 